\title{
The Gerasimov-Drell-Hearn sum rule at MAMI
}

\author{
A. Thomas ${ }^{\mathrm{a}}$ \\ Institut für Kernphysik, Universität Mainz, 55099 Mainz, Germany \\ / \\ Published online: 23 May 2006 - C Società Italiana di Fisica / Springer-Verlag 2006
}

\begin{abstract}
An extended experimental program to investigate the Gerasimov-Drell-Hearn (GDH) sum rule and related partial reaction cross sections on proton and neutron has been carried out by the GDH collaboration at the electron accelerators MAMI (Mainz) and ELSA (Bonn). The GDH sum rule connects the helicity-dependent photoabsorption cross section with the anomalous magnetic moment of the nucleon. The GDH collaboration has measured the total cross section of circularly polarised photons with longitudinally polarised protons at MAMI and ELSA to check this sum rule experimentally. In addition partial reaction channels like pion, double pion and eta production were determined. This provides new information on the helicity-dependent excitation spectrum of the nucleon. With the help of partial wave analyses it is possible to extract new, complementary information on the broad, overlapping resonances in this energy region. The double polarisation observable $E$ measured in this experiment enhances the smaller multipoles via interference terms. The analysis of our data provides a new possibility to study the photon couplings to the nucleon resonances, especially above the $\Delta$ resonance where many properties of the observed states (e.g., coupling constants, branching ratios, helicity amplitudes) are only poorly known. In this paper we present several new results from our measurements on polarised proton and deuteron targets.
\end{abstract}

PACS. 16.60.Le Meson production - 14.20 Baryon resonances with $S=0-25.20$ Photoproduction

\section{Introduction}

The GDH sum rule connects static properties of the nucleon like the anomalous magnetic moment $\kappa$ and the nucleon mass $M$, with the helicity dependent total absorption cross sections $\sigma_{1 / 2}$ and $\sigma_{3 / 2}$, which are related to the dynamics of the excitation spectrum:

$$
\frac{2 \pi^{2} \alpha}{M^{2}} \kappa^{2}=\int_{0}^{\infty} \frac{\mathrm{d} \nu}{\nu}\left(\sigma_{3 / 2}-\sigma_{1 / 2}\right) .
$$

Effectively for the nucleon the lower integration limit is the $\pi$-production threshold energy and $\nu$ denotes the photon energy, $\alpha$ is the fine-structure constant. The GDH sum rule was derived under very general assumptions (Lorentz and gauge invariance, causality, relativity, unitarity and the no-subtraction hypothesis) in 1966 by Gerasimov, Drell and Hearn [1,2], but it was not checked experimentally until the pioneering experiment at MAMI in 1998. Some authors $[3,4]$ have calculated the right hand side of eq. (1) using partial wave analyses of single $\pi$-photoproduction experiments and rough estimates for the double $\pi$ contribution. They always obtained a discrepancy with the left side of eq. (1), which yields $205 \mu \mathrm{b}$ for the proton. The interest in the GDH sum rule was renewed with the measurements of the spin structure functions for proton and neutron, because the GDH sum rule

\footnotetext{
a e-mail: thomas@kph.uni-mainz.de
}

can be seen as an extrapolation from the Bjorken and the Ellis-Jaffe sum rules [5] for deep inelastic lepton scattering to the real photon limit.

Another characteristic property of the nucleon is the forward spin polarisability:

$$
\gamma_{0}=\frac{1}{4 \pi^{2}} \int_{0}^{\infty} \frac{\mathrm{d} \nu}{\nu^{3}}\left(\sigma_{3 / 2}-\sigma_{1 / 2}\right) .
$$

Due to the stronger weighting of the integrand with the third power of the inverse photon energy this integral converges at much lower energies. The predictions for $\gamma_{0}$ by several theoretical calculations reveal serious discrepancies. Especially the results obtained by dispersion relations differ significantly from the predictions of chiral perturbation theory.

Nowadays, improved technologies for polarized photon beams and polarised targets allow us to check this sum rule performing a dedicated double polarisation experiment directly. The goal of the GDH collaboration is to measure the energy dependence of the helicity-dependent total absorption cross section as well as partial reaction channels on proton and neutron targets to determine the dominant contributions to eq. (1). In addition to checking the sum rule experimentally there is a strong motivation to carefully measure the integrand and the helicity dependence of the partial reaction channels such as single- or double-pion photoproduction, which provides completely 


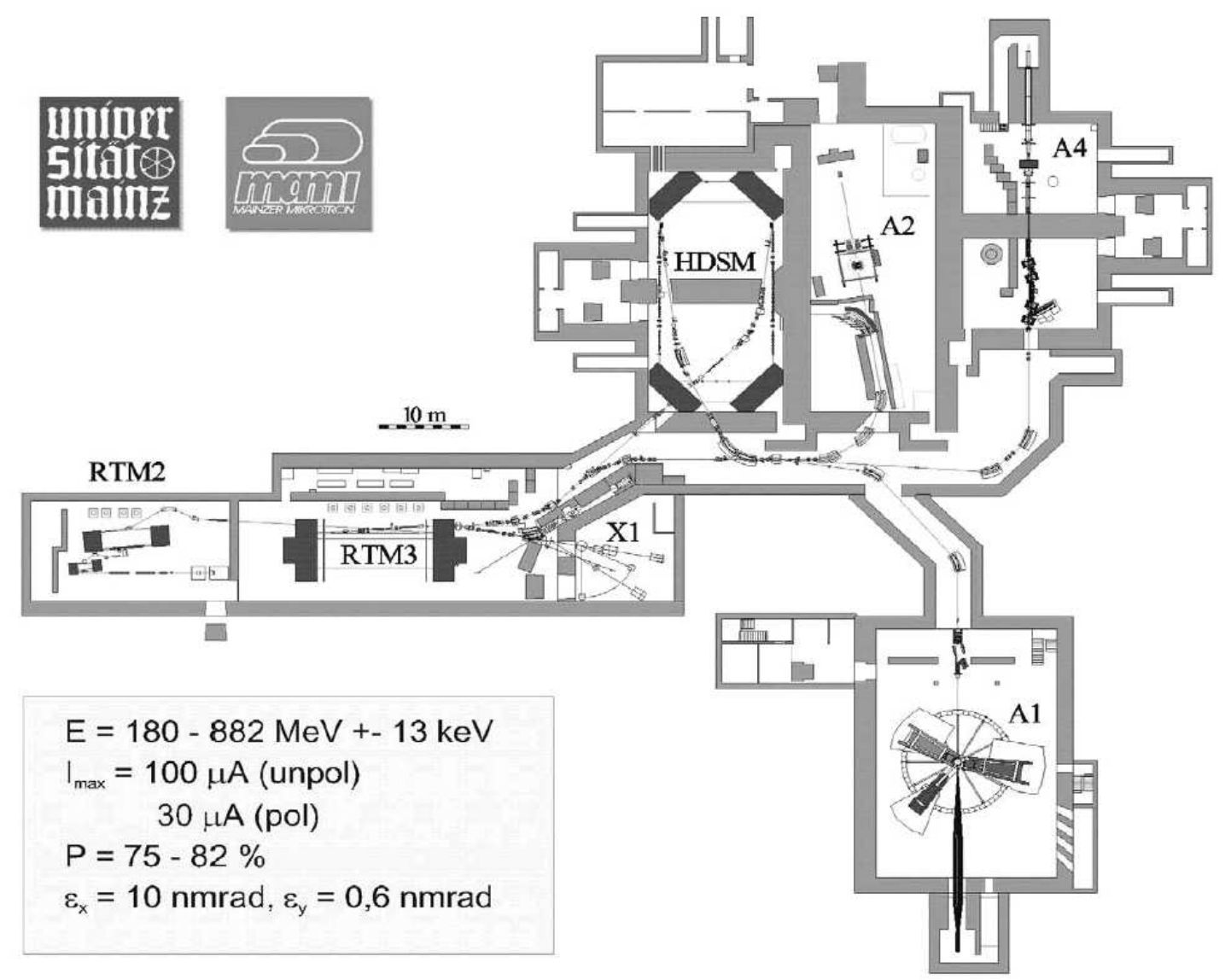

Fig. 1. The Mainz Microtron MAMI. The GDH experiment was performed in the A2 tagger hall.

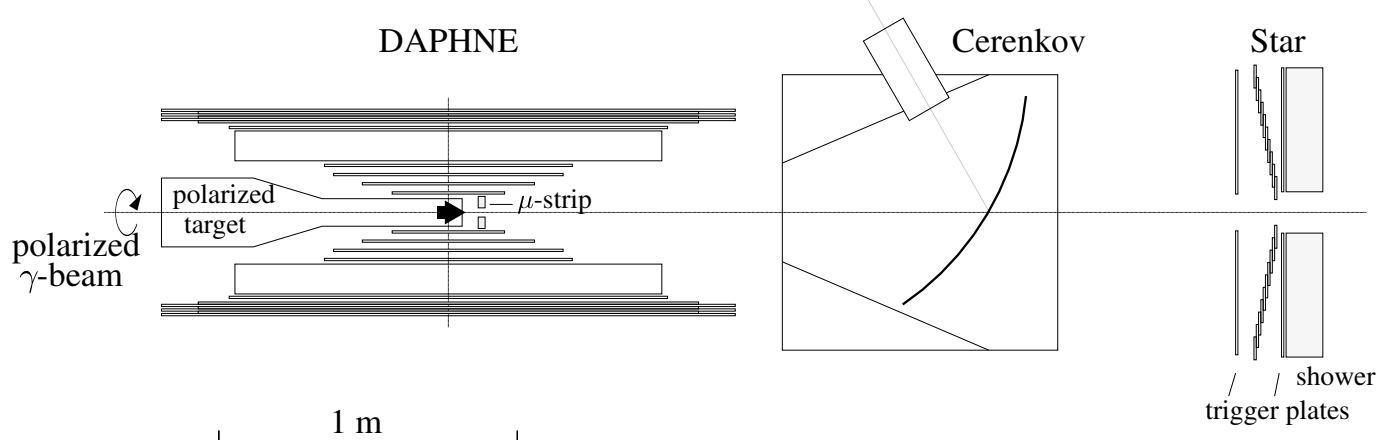

Fig. 2. Overview of the GDH experimental setup at MAMI. The polarised photon beam is coming along the axis of the ${ }^{3} \mathrm{He} /{ }^{4} \mathrm{He}$ refrigerator of the polarised "frozen spin" target.

new and up to now inaccessible information on partialwave amplitudes. Besides the measurements with the polarised proton target, we have also performed an extended series of experiments with polarised deuteron targets in order to extract information on the neutron and thus on the isospin dependence of the helicity structure.

\section{Experimental setup}

The MAMI accelerator (fig. 1) with its source of polarised electrons, based on the photoeffect on a strained GaAs crystal, routinely delivers polarised beams with a maxi- mum energy of $855 \mathrm{MeV}$. In our first successful data taking period in 1998 (fig. 3) we typically had a degree of polarisation of about $75 \%$, in the 2 nd period in the year 2003 for the neutron runs this has been improved to about $80 \%$. A dedicated experimental apparatus (fig. 2) including a polarised solid target and a detector with full angular acceptance was installed in the A2 Hall. The photons were produced by bremsstrahlung in the A2-Glasgow-Mainz tagging facility (fig. 4), which firstly determines the photon energy by the help of 352 scintillation counters with a resolution of approximately $2 \mathrm{MeV}$ at $855 \mathrm{MeV}$ primary beam energy and secondly measures the degree of polarisation 


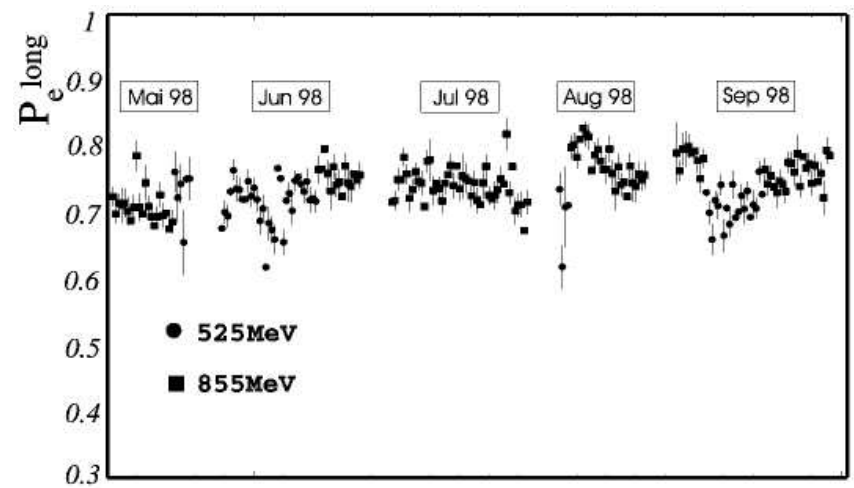

Fig. 3. The time dependence of the electron beam polarisation during the first data-taking period in 1998.
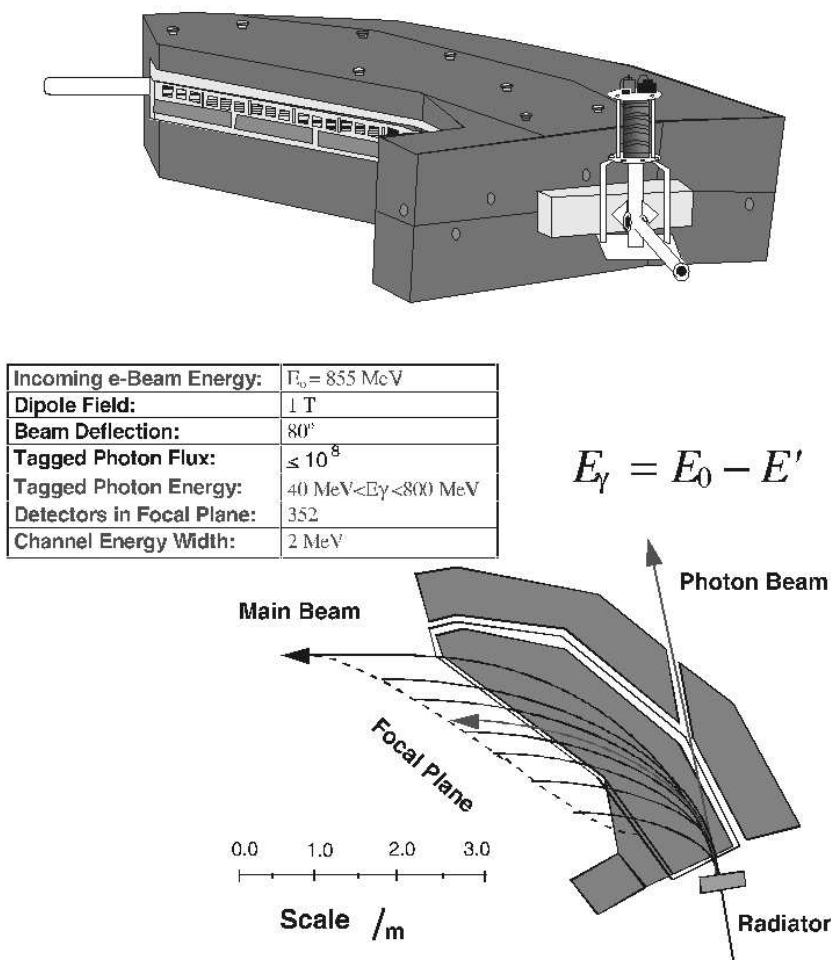

Fig. 4. The A2-Glasgow-Mainz tagging facility.

of the electrons by detecting the asymmetry in the Møller process. This Møller polarimeter allows an online monitoring of the degree of electron beam polarisation. The statistical error is of the order of $2 \%$ for a measuring time of 4 hours (each point in fig. 3 represents 4 hours of data taking).

The energy-dependent helicity transfer to the photon can be calculated reliably [6]. In order to achieve a high degree of photon beam polarisation we used $525 \mathrm{MeV}$ and $855 \mathrm{MeV}$ as primary electron beam energies. Figure 5 shows the degree of circular polarisation of the outgoing photons as function of longitudinal polarisation of the incoming electrons.

A new solid state "frozen spin" polarised target [7], developed and operated by the universities of Bonn, Bochum, Mainz and Nagoya, was used. The tar-

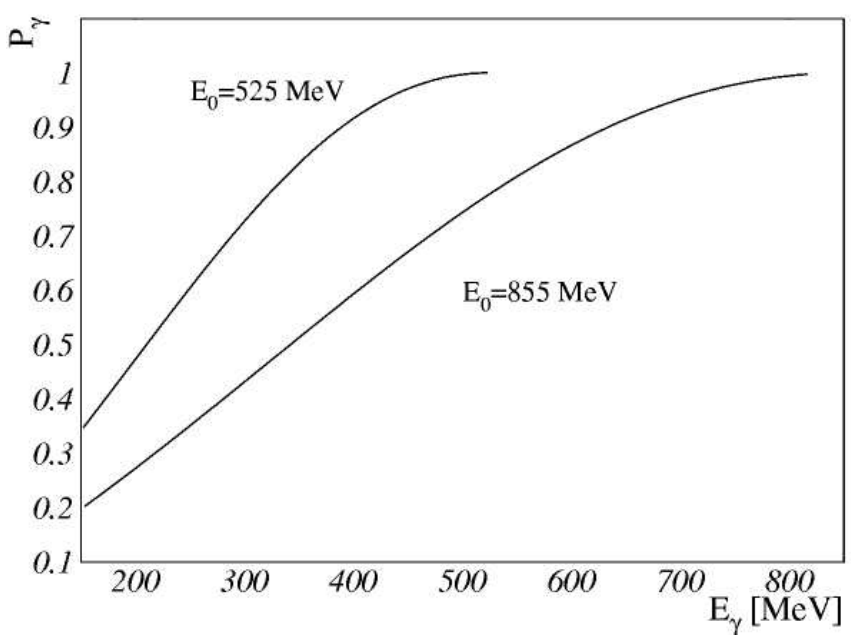

Fig. 5. The helicity transfer from the incoming longitudinal polarised electron to the photons.

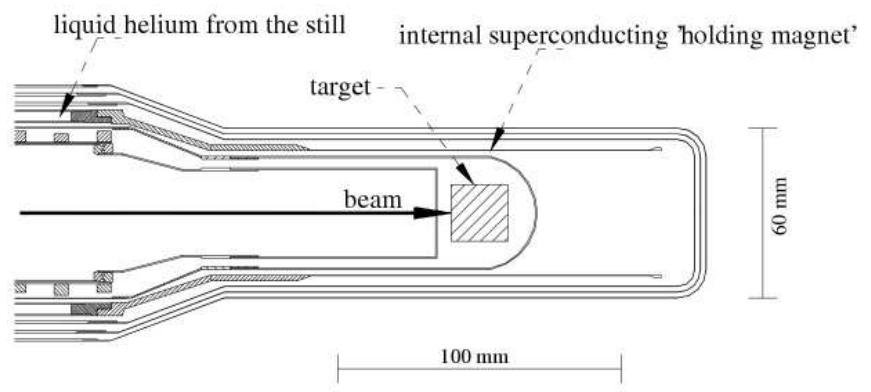

Fig. 6. The end part of the ${ }^{3} \mathrm{He} /{ }^{4} \mathrm{He}$ refrigerator is equipped with a superconduction holding coil.

get materials butanol $\left(\mathrm{C}_{4} \mathrm{H}_{9} \mathrm{OH}\right)$ or deuterated butanol $\left(\mathrm{C}_{4} \mathrm{D}_{9} \mathrm{OD}\right)$, chemically doped with paramagnetic radicals to allow the process of "Dynamic Nuclear Polarisation", were cooled to a temperature of $50 \mathrm{mK}$ in a ${ }^{3} \mathrm{He} /{ }^{4} \mathrm{He}$ dilution refrigerator. In this horizontal cryostat, which was developed and constructed in the Bonn polarised target group, a nearly full angular acceptance was achieved by the integration of a thin internal holding coil on the thermal radiation shields (see fig. 6). This major step forward in polarised target technology was possible by using the inner thermal radiation shield as coil holder and cooling it with liquid ${ }^{3} \mathrm{He} /{ }^{4} \mathrm{He}$ mixture from the still to a temperature of $1.2 \mathrm{~K}$ [8]. The momentum threshold for the outgoing particles is determined by the thickness of the coil including a thermal radiation shield (coil holder), which was equivalent to $0.8 \mathrm{~mm}$ copper.

The polarising magnetic field of $2.5 \mathrm{~T}$ was produced by an external superconducting solenoid. After 4 hours values for the degree of proton polarisation of $80 \%-85 \%$ were reached by irradiation with microwaves of a frequency near to the electron spin resonance $(70 \mathrm{GHz})$. The external solenoid was moved on a rail system and the polarisation was maintained in the "frozen spin" mode at $50 \mathrm{mK}$ and $0.4 \mathrm{~T}$ (see fig. 7). 


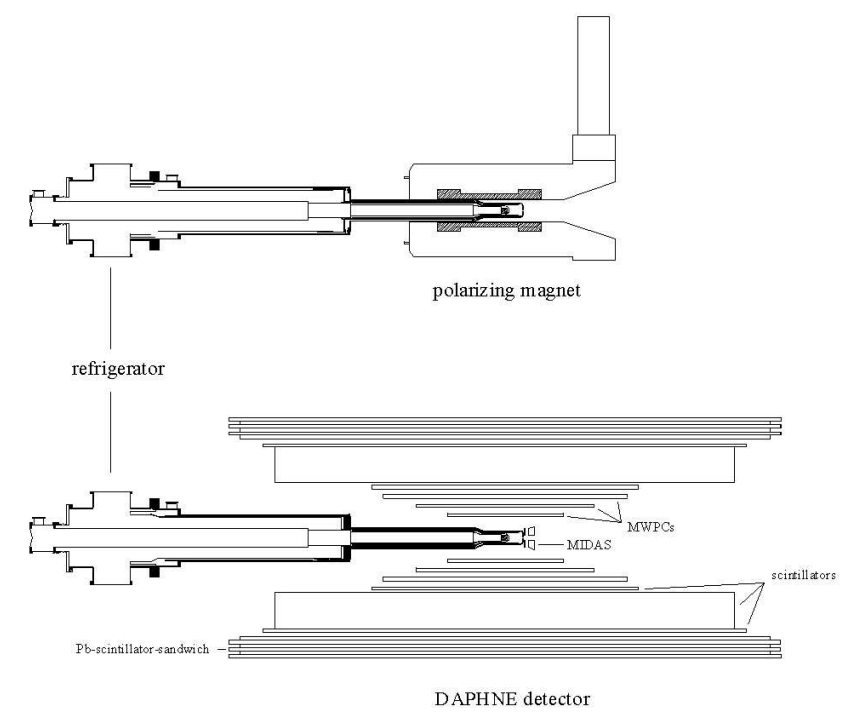

Fig. 7. The experimental principle for the GDH "frozen spin" target. The ${ }^{3} \mathrm{He} /{ }^{4} \mathrm{He}$ refrigerator is stationary, the detector DAPHNE and the polarising solenoid are movable. The upper picture shows the set-up in the polarising mode, the lower one in the data-taking mode. A high-precision rail system was used to move the DAPHNE detector and the superconducting polarising magnet.

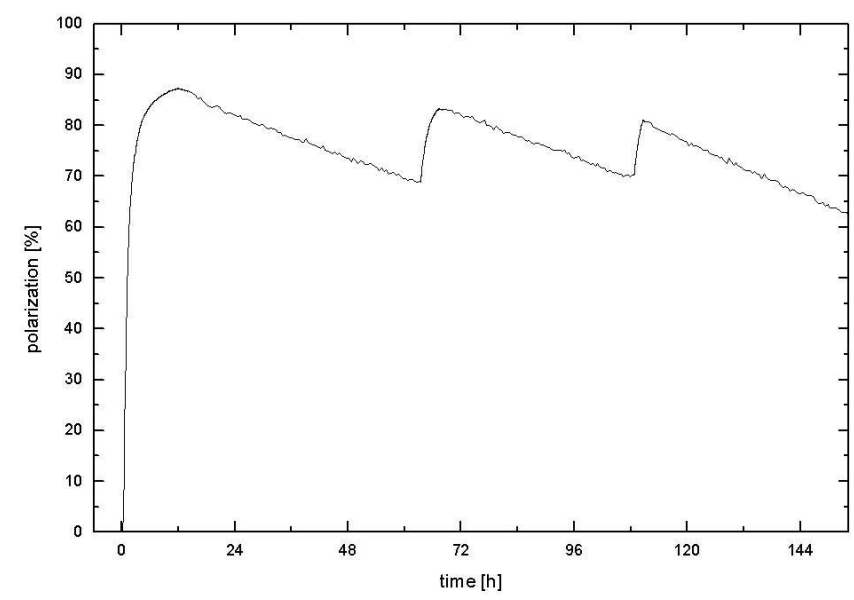

Fig. 8. The time dependence of the target polarisation during the first data-taking period in 1998 .

The relaxation time for the proton spins was about 200 hours. Consequently we could take data for typically 2 days before repolarizing the target, for example three cycles of polarising the target and data taking during the relaxation of the target can be seen in fig. 8. The repolarizing time for the target (approximately 3 hours) was used to refill the 4001 liquid-helium buffer containers for the cryostat and the polarising magnet. The movement of the polarising solenoid and the detector took around 15 minutes.

The cylindrical detector DAPHNE (Détecteur a grande Acceptance pour la Physique photoNucléaire Experimentale) [9] was especially designed for handling multi particle final states by provision of a large solid angle (94\%

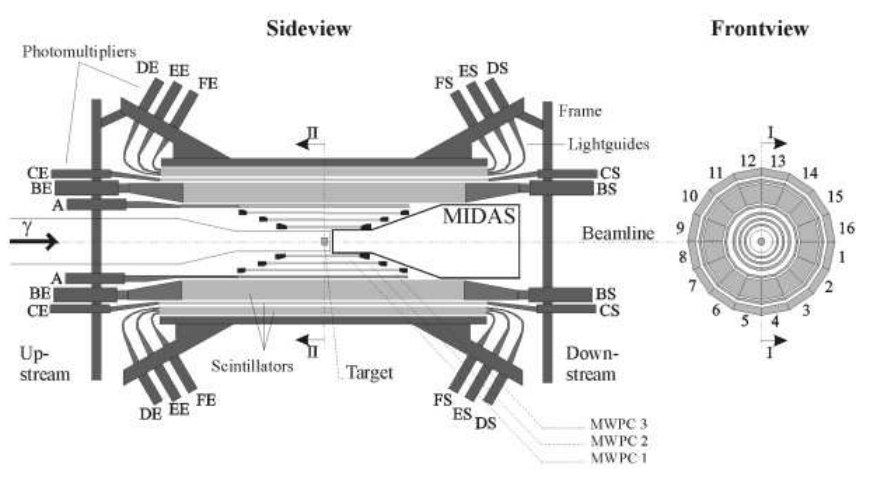

Fig. 9. The DAPHNE detector.

of $4 \pi$ ) particle identification and has a moderate efficiency for neutral particles. DAPHNE was developed by a collaboration of Saclay and Pavia for the investigation of photoreactions on light nuclei. As shown in fig. 9, DAPHNE surrounds the target and has a cylindrical symmetry. It consists of a vertex detector (three multi-wire proportional chambers, MWPC) for reconstructing the trajectories of charged particles surrounded by a hodoscope for their identification and energy determination. The outer part forms a lead-aluminum-scintillator sandwich serving as a calorimeter for the detection of decay photons and protons. Below a primary beam energy of $700 \mathrm{MeV}$ all emitted protons are retained in the detector providing a good energy determination. The threshold momentum for the detection of charged particles emitted from a target including holding coil amounts to $80 \mathrm{MeV} / c$ for pions and $270 \mathrm{MeV} / c$ for protons. Leptonic background in DAPHNE is suppressed by the selection of appropriate discriminator thresholds. In forward direction further detection components (silicon $\mu$-strip detector, a Čerenkov detector, a scintillation counter array, the ring shaped STAR detector) have been added to expand the angular acceptance.

\section{Results}

\subsection{Results on the GDH-Integral and the forward spin polarizibility integral on the proton}

Figure 10 shows our data for the helicity difference of the total cross section measured at MAMI in the first phase of the GDH experiment [10]. The data are compared to predictions from the partial wave analyses SAID and MAID. The negative values of the cross section difference $\left(\sigma_{3 / 2}-\sigma_{1 / 2}\right)$ in the threshold region are due to the dominance of the $E_{0+}$ multipole in the single $\pi^{+}$production channel. The excitation of the $\Delta$-resonance $\left(P_{33}(1232)\right)$ prefers the $\sigma_{3 / 2}$ cross section at energies around $300 \mathrm{MeV}$. It is excited by a strong $M_{1+}$ transition with only $2.5 \%$ $E_{1+}$ admixture. At higher energies the double pion production plays an important role and is not represented in the theoretical curves.

In fig. 11 the experimental running GDH integral (right-hand side of eq. (1)) is displayed and compared to the model predictions. The integration starts at 


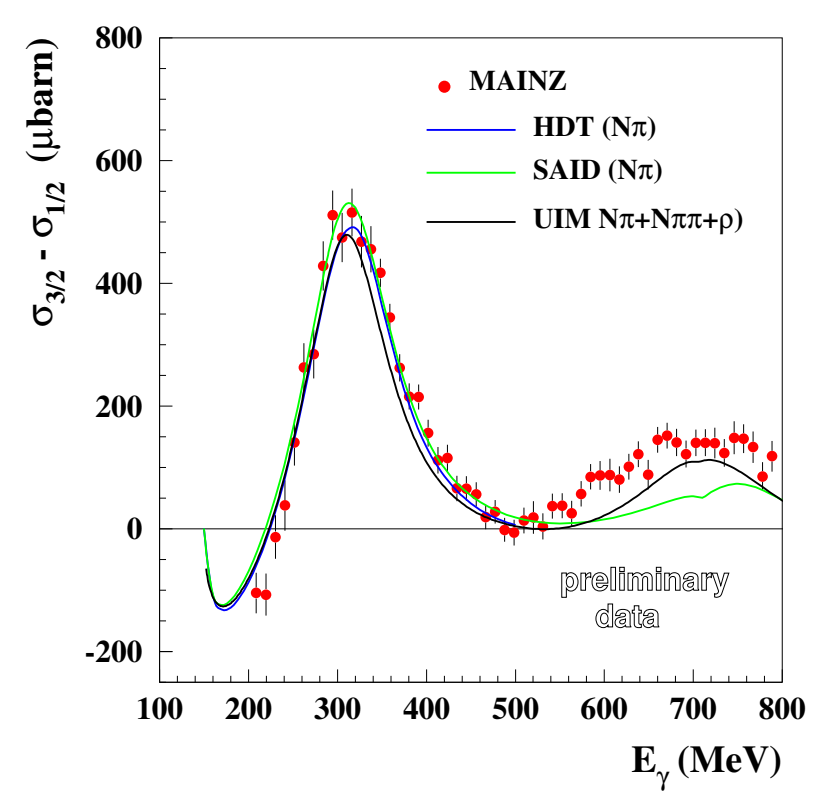

Fig. 10. The total cross section difference $\left(\sigma_{3 / 2}-\sigma_{1 / 2}\right)$ on ${ }^{1} \mathrm{H}$ is compared to previous results [11] (open circles) and to the predictions of the HDT [12], SAID [13] and UIM [14] analyses. Only statistical errors are shown.

Table 1. Measured values of the GDH integral in various photon energy intervals (see text for references).

\begin{tabular}{|r|l|l|}
\hline \hline & $\nu[\mathrm{GeV}]$ & GDH Integral $[\mu \mathrm{b}]$ \\
\hline ELSA & $0.8-2.9$ & $27.5 \pm 2.0 \pm 1.2$ \\
MAMI & $0.2-0.8$ & $226 \pm 5 \pm 12$ \\
\hline combined & $0.2-2.9$ & $253.5 \pm 5 \pm 12$ \\
\hline \hline
\end{tabular}

Table 2. Measured value of the GDH integral and model predictions for the unmeasured energy intervals (see text for references).

\begin{tabular}{|r|l|l|}
\hline \hline & $\nu[\mathrm{GeV}]$ & GDH Integral $[\mu \mathrm{b}]$ \\
\hline MAID & $<0.2$ & -27.5 \\
SAID & $<0.2$ & -28 \\
Exp.(MAMI+ELSA) & $0.2-2.9$ & $253.5 \pm 5 \pm 12$ \\
Simula et al. & $>2.9$ & -13 \\
Bianchi and Thomas & $>2.9$ & -14 \\
\hline combined & & $211.5-213$ \\
GDH sum rule value & & 205 \\
\hline \hline
\end{tabular}

$E_{\gamma}=200 \mathrm{MeV}$ and the upper integration limit is taken as the running variable. The measured value of the GDH integral between 200 and $800 \mathrm{MeV}$ amounts to $226 \pm$ 5 (stat) \pm 12 (sys) $\mu \mathrm{b}$.

Due to the $\nu^{-3}$ weighting in eq. (2), the $\gamma_{0}$ running integral is almost saturated at $E_{\gamma}=800 \mathrm{MeV}$. The value of the $\gamma_{0}$ integral between 200 and $800 \mathrm{MeV}$ amounts to $[-187 \pm 8($ stat $) \pm 10($ sys $)] \cdot 10^{-6} \mathrm{fm}^{4}$.

The data taken at ELSA $[15,16]$ from 0.7 to $2.9 \mathrm{GeV}$ (see fig. 12), together with the previously measured data at MAMI, cover a broader photon energy interval, and result

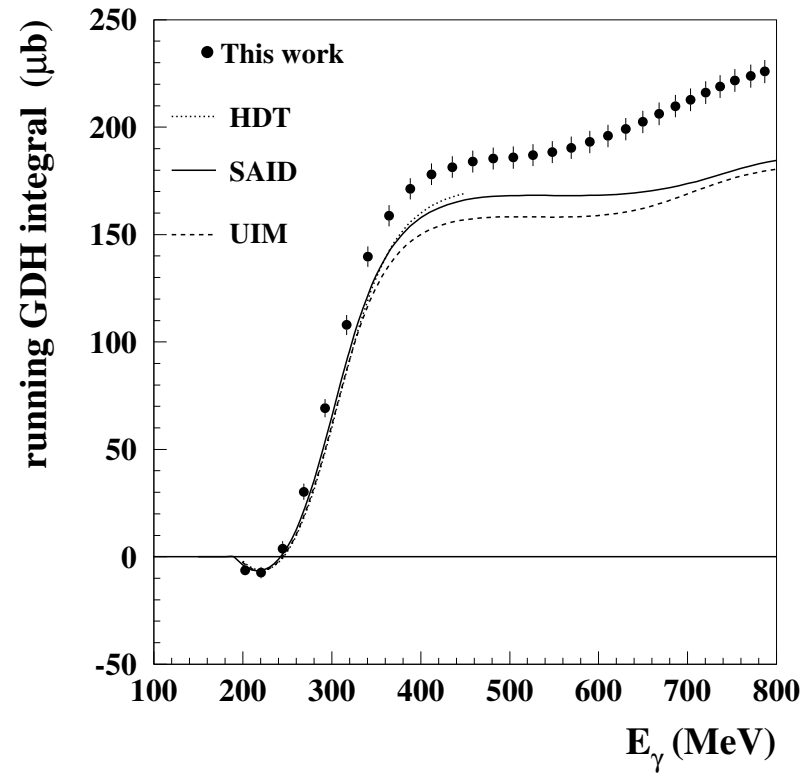

Fig. 11. The running GDH integral obtained in this work starting at $200 \mathrm{MeV}$ is compared to the model predictions. Only statistical errors are shown.

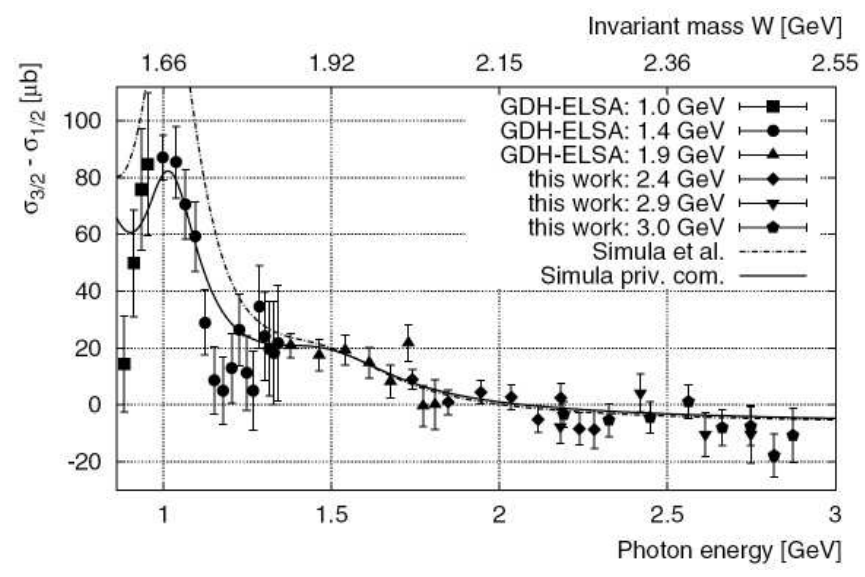

Fig. 12. The helicity dependent total photoabsorption cross section difference in the second and third resonance region measured at ELSA.

in $254 \mu \mathrm{b}$ for the GDH integral between 0.2 and $2.9 \mathrm{GeV}$, see table 1 . In order to check the validity of the GDH sum rule the contribution of the missing low and high energy regions have to be added according to the existing models (see table 2): The unitary isobar model MAID2002 [14] gives a contribution of $(-27.5 \pm 3) \mu \mathrm{b}$ for photon energies below $0.20 \mathrm{GeV}$ [17]. The Regge approaches from $[18,19]$ predict a negative contribution above $2.9 \mathrm{GeV}$ of $-14 \mu \mathrm{b}$ and $-13 \mu \mathrm{b}$, respectively. The combination of our experimental results from MAMI and ELSA with these predictions yields an estimate of $(211.5-213) \mu \mathrm{b}$ which within the experimental errors of \pm 5 (stat) \pm 12 (sys) $\mu \mathrm{b}$ is consistent with the GDH sum rule value of $205 \mu \mathrm{b}$. 


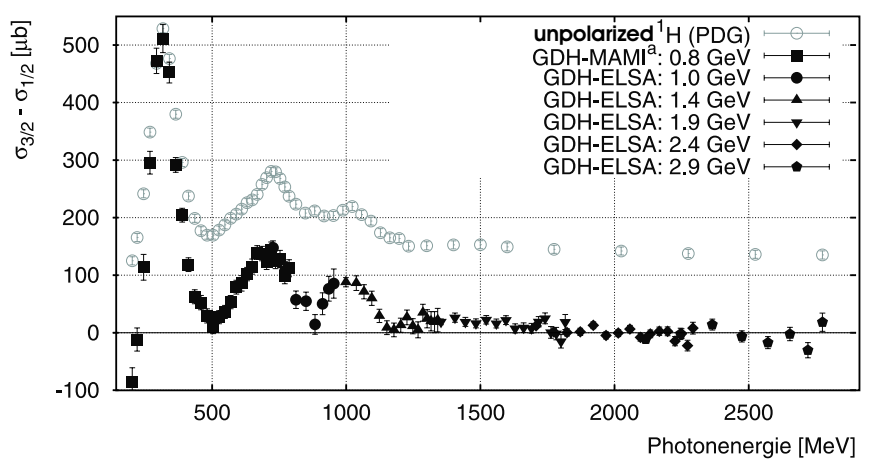

Fig. 13. Helicity difference $\Delta \sigma=\sigma_{3 / 2}-\sigma_{1 / 2}$ and unpolarised total photoproduction cross section on the proton (combined data set from MAMI and ELSA).

A summary of the present status is shown in fig. 13, where the helicity difference $\Delta \sigma=\sigma_{3 / 2}-\sigma_{1 / 2}$ for the total cross section on the proton is compared to the unpolarised cross section. As the large background of non-resonant photoproduction is spin independent and therefore almost disappears in $\Delta \sigma$, the helicity difference delivers valuable information to study the properties of nucleon resonances.

\subsection{Results on partial reaction channels}

The DAPHNE detector with forward components was capable to measure besides the total absorption cross section also partial reaction channels (see fig. 14). We have published our results on the proton in a series of papers:

- the total photoabsorption cross section for energies up to $800 \mathrm{MeV}$ [11]

- the single pion production channels up to a photon energy of $450 \mathrm{MeV}[10]$ and [20]

- the single $\pi^{0}$ production up to a photon energy of $800 \mathrm{MeV}[21]$

- the single $\pi^{+}$production up to a photon energy of $800 \mathrm{MeV}[22]$

- the double pion production channels $\pi^{0} \pi^{+}[23]$ and $\pi^{0} \pi^{0}[24]$

- helicity dependent $\eta$ production [25].

Besides the importance of this data to check the GDH sum rule and to measure the forward spin polarizibility as shown in the previous section, new information about the nucleon's excitation spectrum can be extracted. This feature can be understood from the multipole decomposition of the $\gamma N \rightarrow N \pi$ total cross section. In the following we use the pion multipole notation, where $E$ and $M$ denote the electric or magnetic character of the incoming photon and the indices $l_{ \pm}$describe the coupling of the pion angular momentum $l$ and the nucleon spin to the total angular momentum $J=l \pm 1 / 2$. In the $\Delta(1232)$-resonance region the measured helicity asymmetry is sensitive to the ratio of the multipoles $E M R=\frac{E_{1+}}{M_{1+}}$. In the second resonance region $(500 \mathrm{MeV} \leq \nu \leq 900 \mathrm{MeV})$, where several overlapping states are present, e.g., $P_{11}(1440), D_{13}(1520)$, $S_{11}(1535)$, the helicity dependent observables are particularly sensitive to the behaviour of the electromagnetic

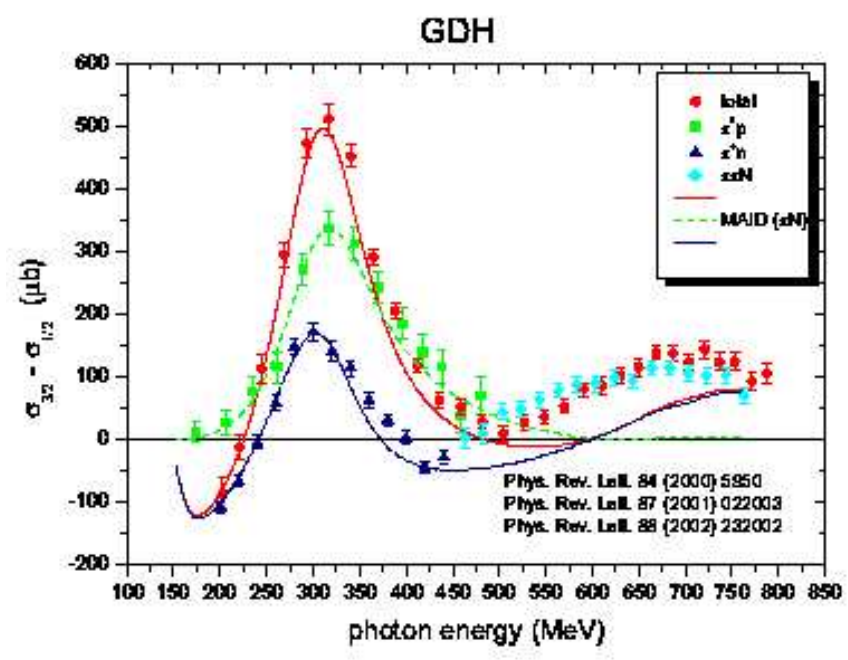

Fig. 14. Overview on the helicity dependent partial photoabsorption cross sections measured at MAMI.

multipoles which are responsible for the excitation of the $D_{13}(1520)$-resonance.

Considering only partial waves with $l \leq 2$, the total unpolarised cross section $(\sigma)$ can then be written as (see, for instance, [26]):

$$
\begin{aligned}
\sigma \propto\left|E_{0+}\right|^{2}+\left|M_{1-}\right|^{2}+6\left|E_{1+}\right|^{2}+ \\
\quad 2\left|M_{1+}\right|^{2}+6\left|M_{2-}\right|^{2}+2\left|E_{2-}\right|^{2}+\ldots,
\end{aligned}
$$

while the helicity dependent total cross section $\Delta \sigma_{31}=$ $\left(\sigma_{3 / 2}-\sigma_{1 / 2}\right)$, where the subscripts $3 / 2(1 / 2)$ correspond to the (anti)parallel $\gamma$-nucleon spin configuration, is

$$
\begin{array}{r}
\Delta \sigma_{31} \propto-\left|E_{0+}\right|^{2}-\left|M_{1-}\right|^{2}-3\left|E_{1+}\right|^{2}+\left|M_{1+}\right|^{2}- \\
6 E_{1+}^{*} M_{1+}-3\left|M_{2-}\right|^{2}+\left|E_{2-}\right|^{2}+6 E_{2-}^{*} M_{2-}+\ldots
\end{array}
$$

Since the unpolarised cross section $\sigma$ of eq. (3) is given by the sum of absolute squares of multipoles, only a few dominant partial waves can be confidently evaluated. On the other hand, the sensitivity to some the weaker multipoles is greatly enhanced by measuring the polarised cross section $\Delta \sigma_{31}$ of eq. (4). In particular new interference terms appear, e.g., between $E_{1+}$ and $M_{1+}$, which are directly related to the $\Delta(1232)$ or $P_{33}(1232)$ excitation in the lowenergy region or between $E_{2-}$ and $M_{2-}$, which are directly related to the $D_{13}(1520)$ excitation.

\subsection{The $\Delta(1232)$-resonance region}

There has been an extended program in different laboratories to increase our knowledge about the EMR ratio for the $\Delta(1232)$-resonance $[27,28]$. The determination of the double-polarisation observable E provides new, complementary information to clarify this question. In figs. 15 and 16 we have compared our data with predictions from the multipole analysis MAID2000 using EMR values of $-2.5 \%, 0 \%$ and $+2.5 \%$. The data are well reproduced with an $E M R=-2.5 \%$ [20]. 


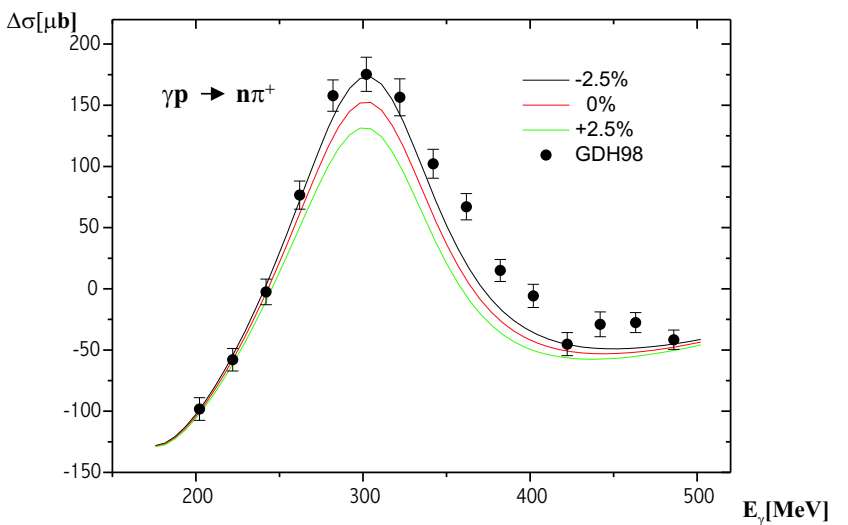

Fig. 15. Sensitivity of the GDH observable $\sigma_{3 / 2}-\sigma_{1 / 2}$ for the EMR ratio of the $\Delta(1232)$ resonance for single $\pi^{+}$production. The curves were produced using MAID2000.

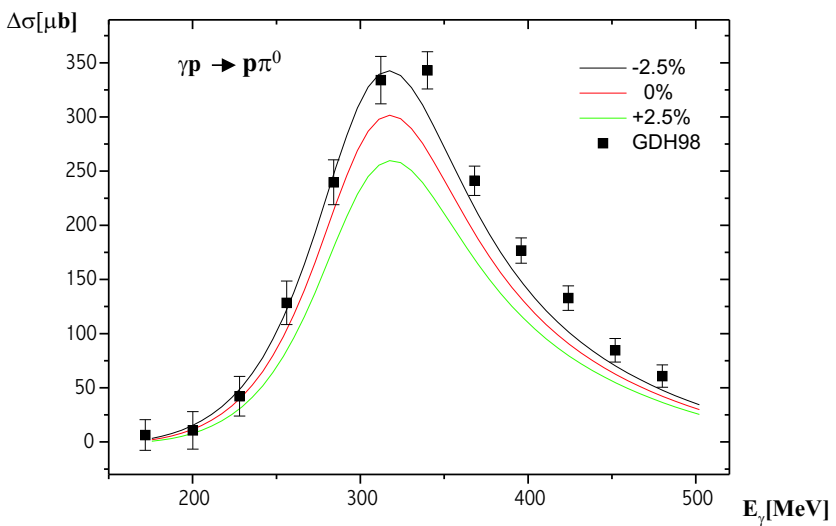

Fig. 16. Sensitivity of the GDH observable $\sigma_{3 / 2}-\sigma_{1 / 2}$ for the EMR ratio of the $\Delta(1232)$ resonance for single $\pi^{0}$ production. The curves were produced using MAID2000.

Due to its cylindrical symmetry the DAPHNE detector is ideally suited to measure angular distributions of the outgoing particles, see for example figs. 17 and 18 for $\pi^{+}$ production [20]. Consequently the angular dependence of the new double polarisation observable has delivered urgently required input for partial wave analyses.

\subsection{The second resonance region}

Since DAPHNE also has a moderate efficiency for neutral particle detection we can distinguish in our data all the contributing partial reaction channels in the 2 nd resonance region. Figure 19 shows our results for the single $\pi^{0}$ production. The agreement of the MAID2000 partial-wave analysis with our data could be improved significantly by changing the parametrisation of the multipoles $E_{2-}$ and $M_{2-}$ that drive the $D_{13}$-excitation by approximately $20 \%$.

In addition fig. 20 shows our preliminary results [29, 22 ] for single $\pi^{+}$production at higher energies. Although the $\pi^{+}$channel is mainly produced via intermediate $D_{13}(1520)$ excitation, a cusp structure can be observed close to the $\eta$ production threshold in the $\sigma_{1 / 2}$ channel.

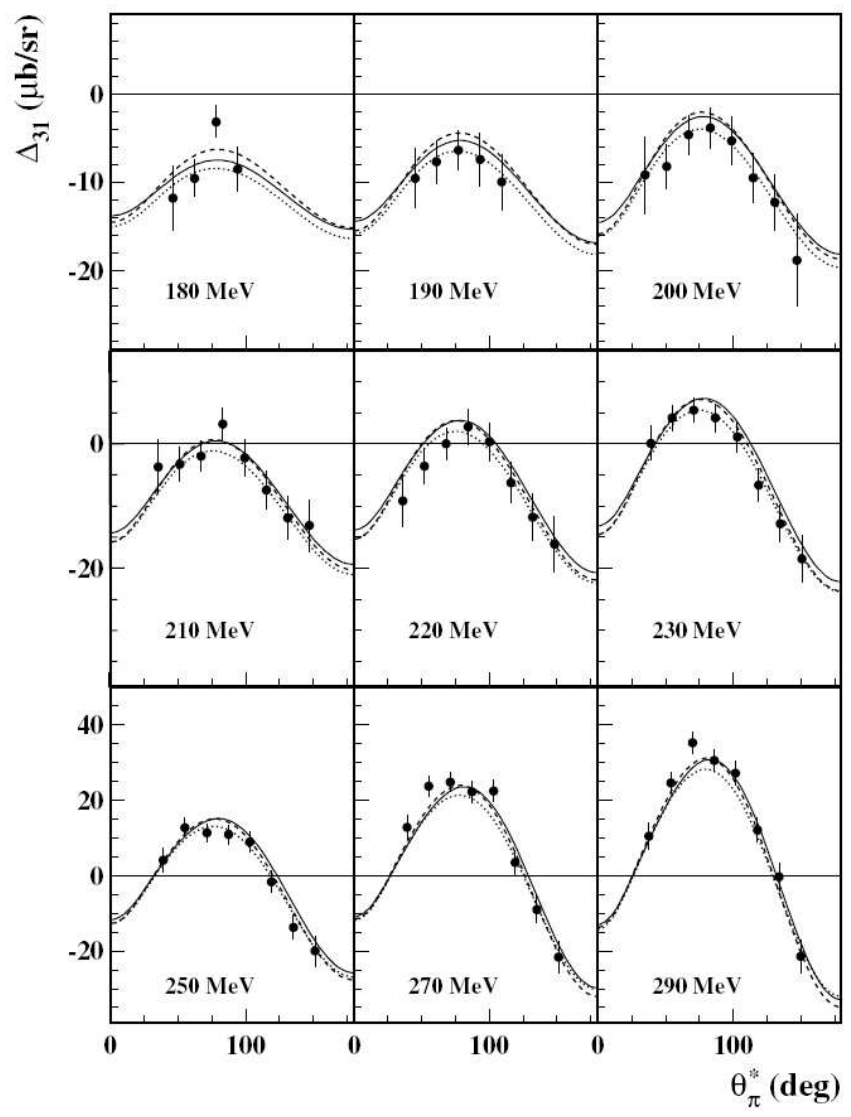

Fig. 17. Angular dependency for the GDH observable $\Delta_{31}=$ $\sigma_{3 / 2}-\sigma_{1 / 2}$ for single $\pi^{+}$production from detection threshold to $290 \mathrm{MeV}$ incoming photon energy. The curves were produced using the MAID and SAID partial wave analyses.

Combining the data from our double polarised experiment with the new beam asymmetry data from Grenoble and Yerewan will improve the knowledge on the higher, strongly overlapping resonances. A detailed discussion can be found in $[21,25,22]$.

\subsection{Double-pion production}

Additional channels are needed to disentangle the resonances at higher nucleon excitation energies. Double pion photoproduction is particularly important for the study of the second resonance region, where the $P_{11}(1440)$ state with its unclear origin is located, since almost $50 \%$ of the total photoabsorption cross section can be attributed to the $N \pi \pi$ channels. In the MAMI B energy range up to $800 \mathrm{MeV}$ we are presently analysing our data for the helicity difference $\sigma_{3 / 2}-\sigma_{1 / 2}$ of the double-pion production channel $\pi^{+} \pi^{-}$. The results for the $\pi^{+} \pi^{0}$ and $\pi^{0} \pi^{0}$ channels have already been published [23,24]. Comparing our results with predictions from different theoretical models $[30,31]$ gives a new insight into the double pion production mechanism, specially the role of the $D_{13}(1520)$, the $P_{11}(1440)$ and the $\Delta(1700)$ are under discussion. 


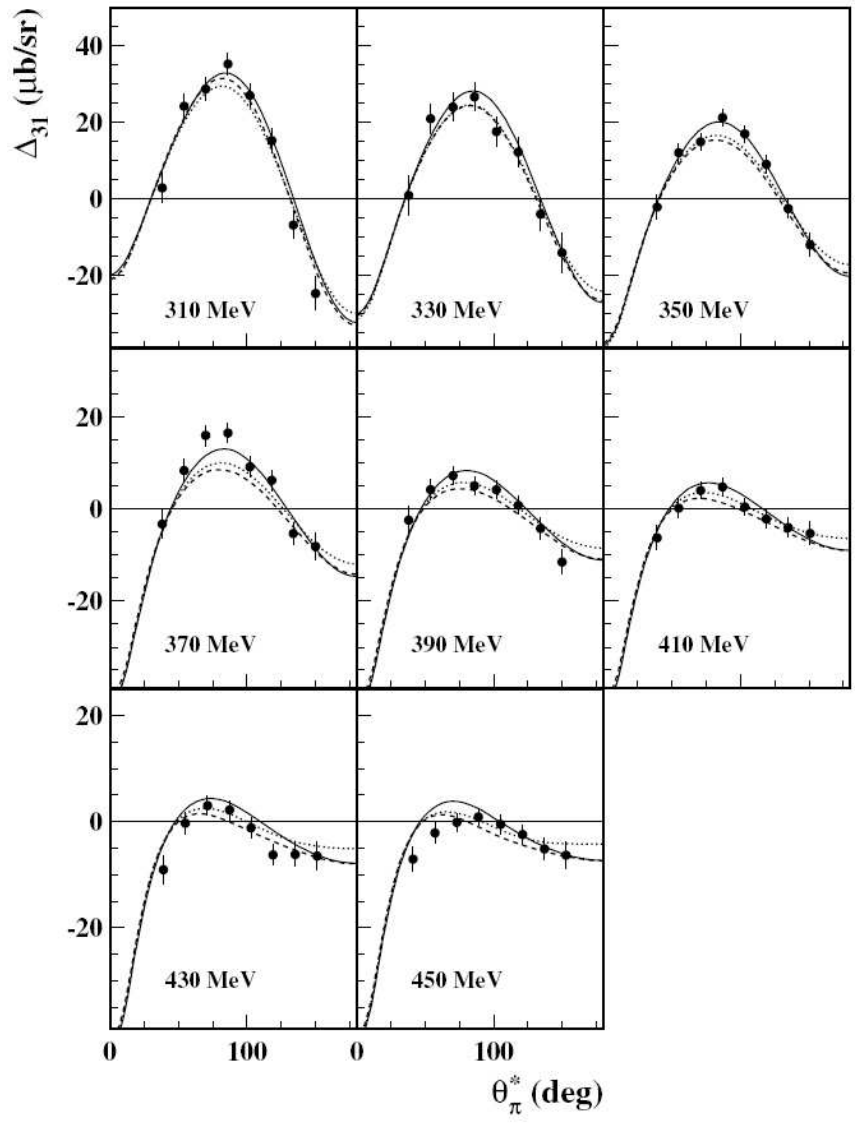

Fig. 18. Angular dependency for the GDH observable $\Delta_{31}=$ $\sigma_{3 / 2}-\sigma_{1 / 2}$ for single $\pi^{+}$production for photon energies between $300 \mathrm{MeV}$ and $450 \mathrm{MeV}$. The curves were produced using the MAID and SAID partial wave analyses.

A strong helicity dependence can be observed in our data (see figs. 21 and 22), with a clear dominance of the $\sigma_{3 / 2}$ over the $\sigma_{1 / 2}$ cross section. This suggests a resonant behaviour due to the intermediate excitation of the $D_{13}(1520)$-resonance. However, the $\sigma_{1 / 2}$ cross section is not negligible, with an indication of a resonance contribution, possibly from the $P_{11}(1440)$ excitation. Future experiments with the recently installed Crystal Ball detector with its high efficiency for neutral particles will deliver further insights in the production mechanism.

\section{6 $\eta$ photoproduction}

The helicity dependence of the $\eta$ production has been measured for the first time at a center-of-mass angle of $\Theta_{\eta}^{*}=70^{\circ}$ in the proton energy range from $780 \mathrm{MeV}$ to $790 \mathrm{MeV}$ and was reported in [25].

The results shown in fig. 23 demonstrate the importance of the $S_{11}(1535)$ resonance for $\eta$ production. The cross section $\left(\frac{\mathrm{d} \sigma}{\mathrm{d} \Omega}\right)_{3 / 2}$ is small as expected for $S$-wave dominance near threshold. Clearly, better statistics and a wider kinematical range are required to disentangle the small contributions from resonances other than the $S_{11}(1535)$.

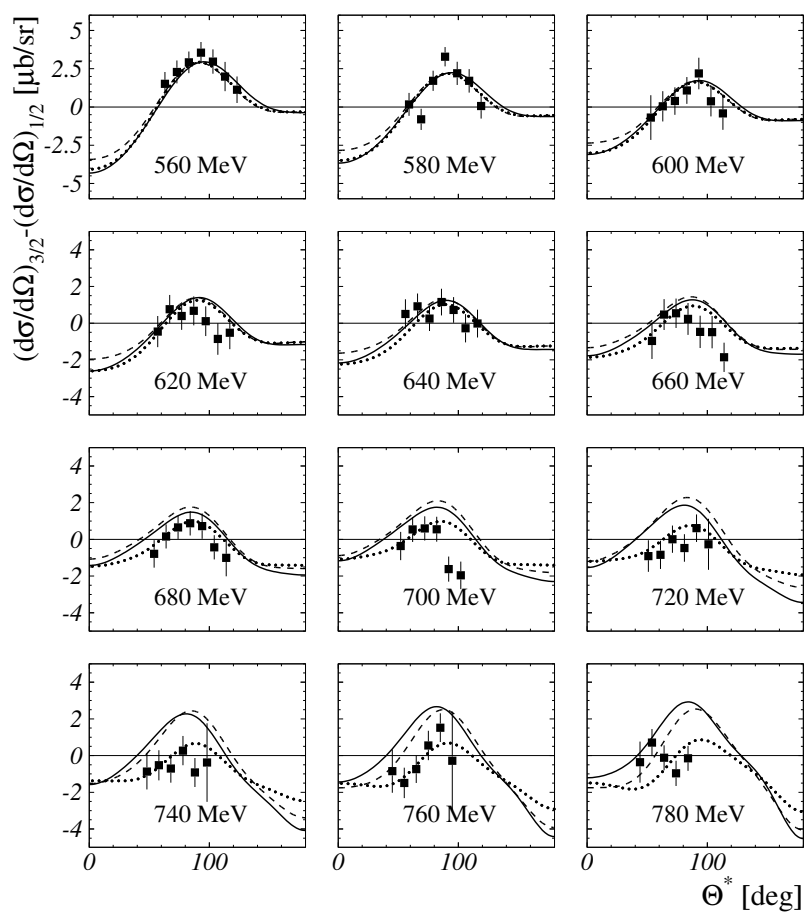

Fig. 19. The asymmetry $\sigma_{3 / 2}-\sigma_{1 / 2}$ for selected energies for single $\pi^{0}$ production compared to the MAID2000 (black) and SAID SP01 (grey) analyses. The dashed curve was produced using MAID2000 with a modified parametrisation for the $D_{13}(1520)$-resonance.

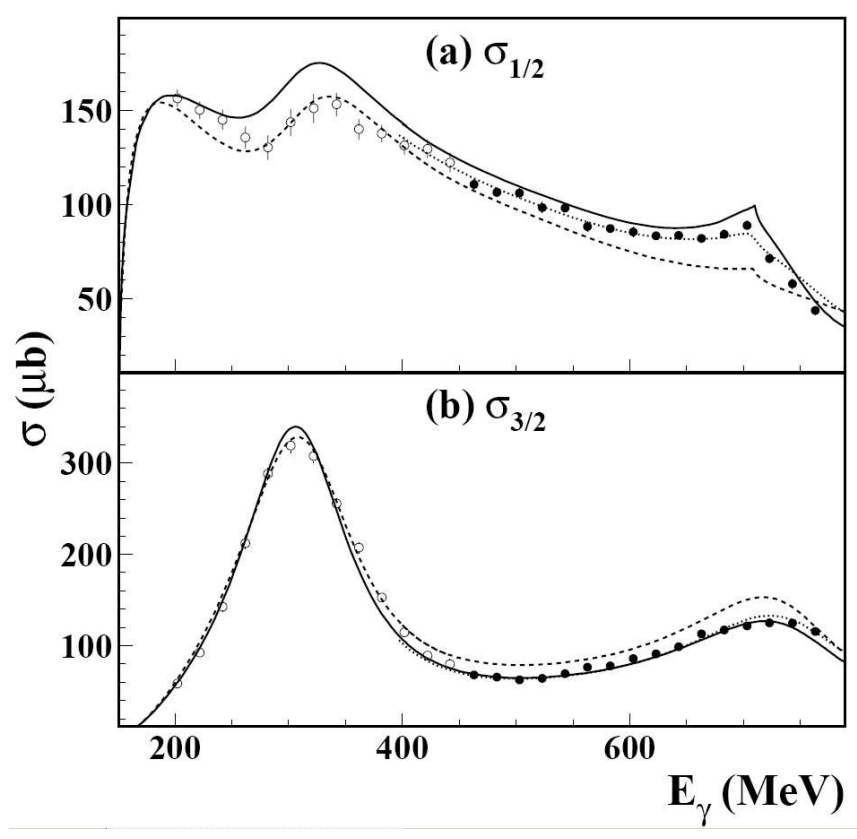

Fig. 20. Preliminary data for $\sigma_{3 / 2}$ and $\sigma_{1 / 2}$ for $\pi^{+}$production. A pronounced cusp structure due to an interference with the $\eta$ production process at threshold can be clearly observed in the $\sigma_{1 / 2}$ cross section [22]. 


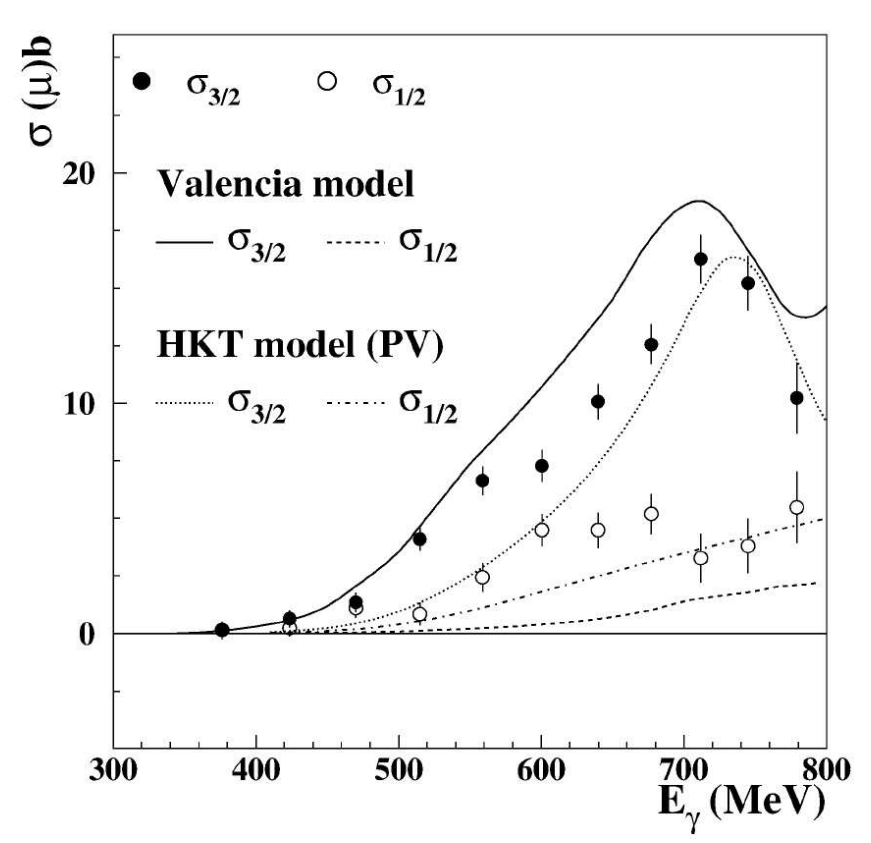

Fig. 21. The helicity dependent cross-sections for $\pi^{0} \pi^{0}$ photoproduction. The errors shown are statistical only.

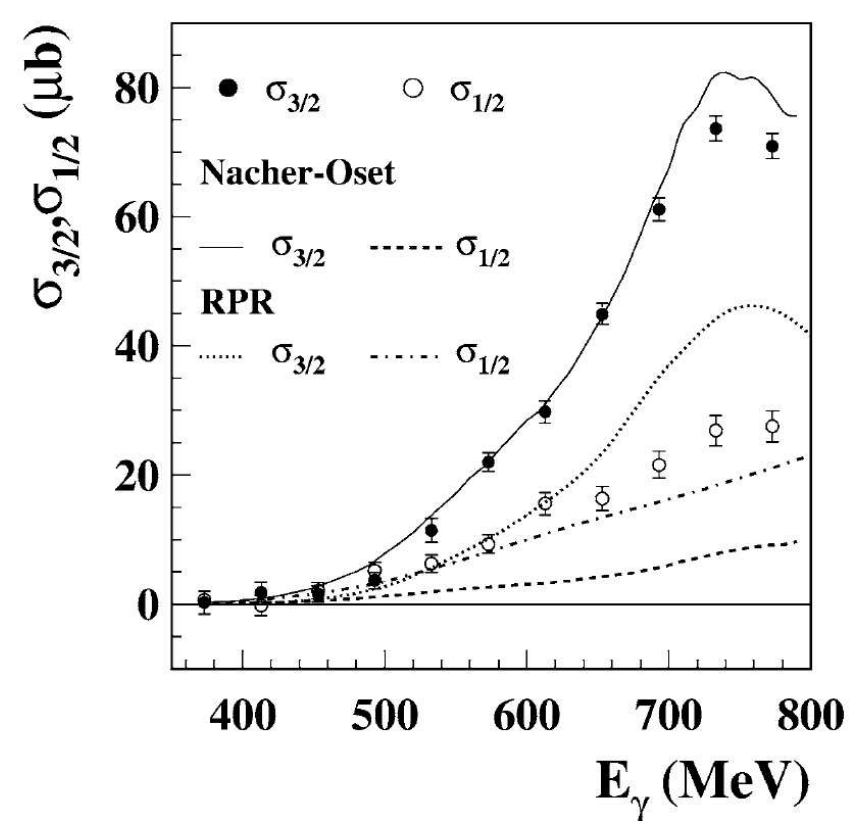

Fig. 22. The helicity dependent differential cross-sections for $\pi^{+} \pi^{0}$ photoproduction. The errors shown are statistical only.

With the new accelerator stage MAMI C and the multiphoton detector Crystal Ball in combination with TAPS as forward detector the data set will be improved significantly.

\subsection{Results on the GDH sum rule on the neutron}

In 1998 there has been done a pilot experiment to investigate the feasibility to measure the GDH sum rule for
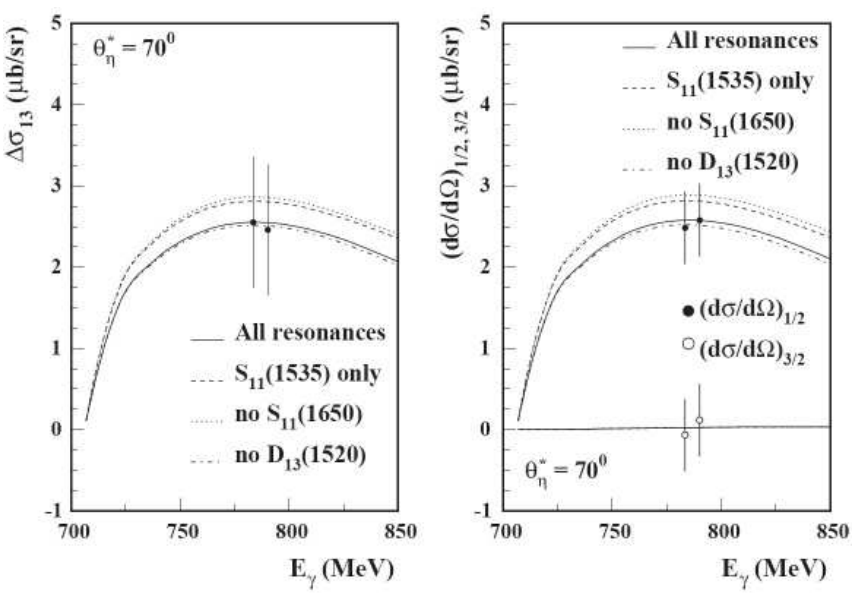

Fig. 23. The measured helicity dependent differential crosssections for $\eta$ photoproduction. Left: $\Delta \sigma_{13}=\left(\frac{\mathrm{d} \sigma}{\mathrm{d} \Omega}\right)_{1 / 2}-$ $\left(\frac{\mathrm{d} \sigma}{\mathrm{d} \Omega}\right)_{3 / 2}$. Right: $\left(\frac{\mathrm{d} \sigma}{\mathrm{d} \Omega}\right)_{1 / 2}$ and $\left(\frac{\mathrm{d} \sigma}{\mathrm{d} \Omega}\right)_{3 / 2}$. The different lines show the predictions of the MAID analysis for four different cases including all resonances (continuous line), $S_{11}(1535)$ only (dashed line), without $S_{13}(1520)$ (dash-dotted line), without $S_{11}(1650)$ (dotted line). The errors shown are statistical only.

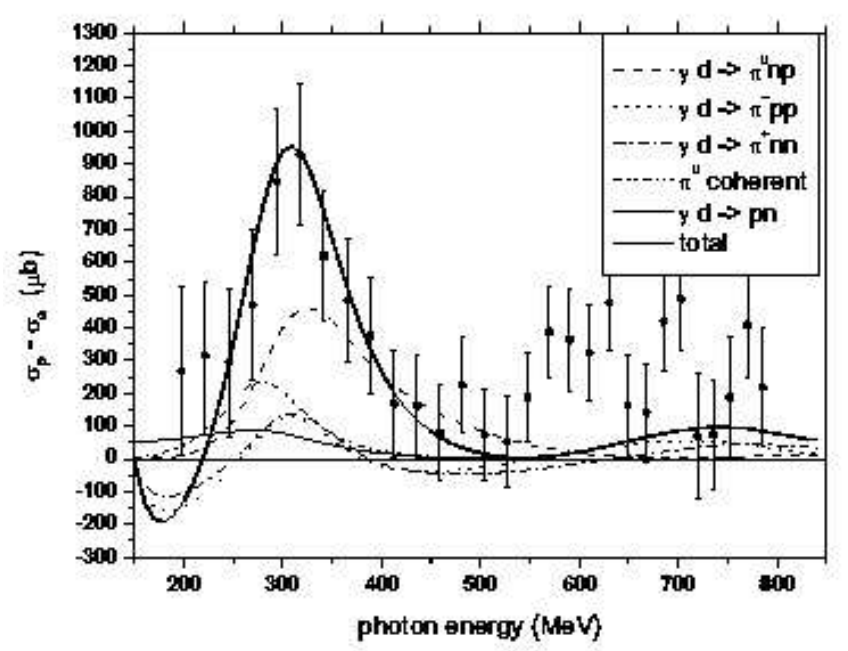

Fig. 24. Preliminary helicity-dependent total cross section for the deuteron compared to theoretical predictions from ref. [32, $33,34]$.

the neutron. Since there is no free neutron target available, the Bonn frozen spin target had to be loaded with deuterated butanol $\left(\mathrm{C}_{4} \mathrm{D}_{9} \mathrm{OD}\right)$.

First preliminary results are available for the deuteron for the total cross section (see fig. 24) and the partial channels $\vec{\gamma} \vec{d} \rightarrow p p \pi^{-}$and $\vec{\gamma} \vec{d} \rightarrow n n \pi^{+}$[35], based on a small subset of the data.

The full data taking was carried out in the first half of 2003 using the Bonn frozen-spin target [7]. In the course of the experiment the degree of polarisation could be increased from $35 \%$ to more than $70 \%$ due to a new target material based on trityl-doped D-butanol [36]. This development by the Bochum polarised target group is a major 


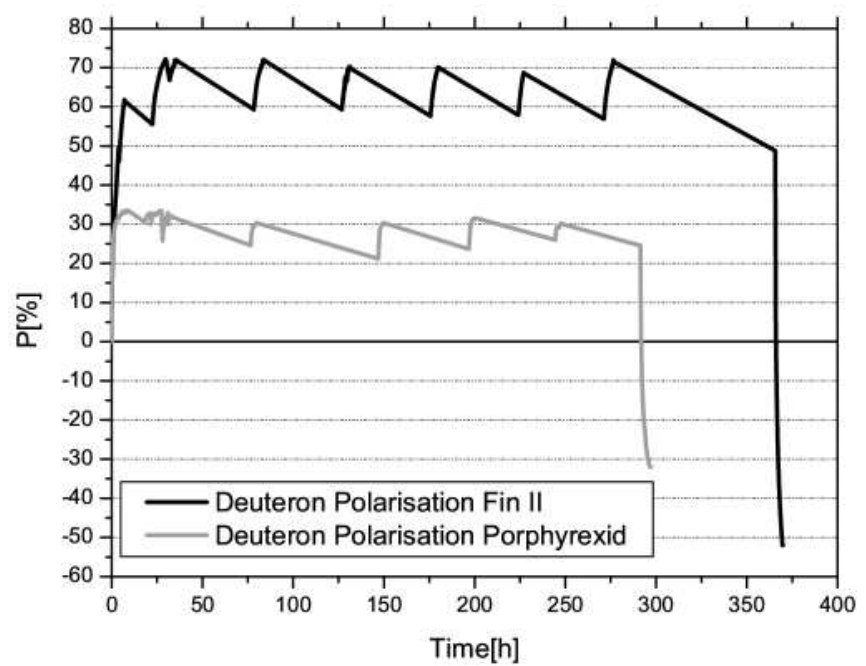

Fig. 25. Progression of the target polarisation during the experiment with the standard and the new target material (labeled Fin II).

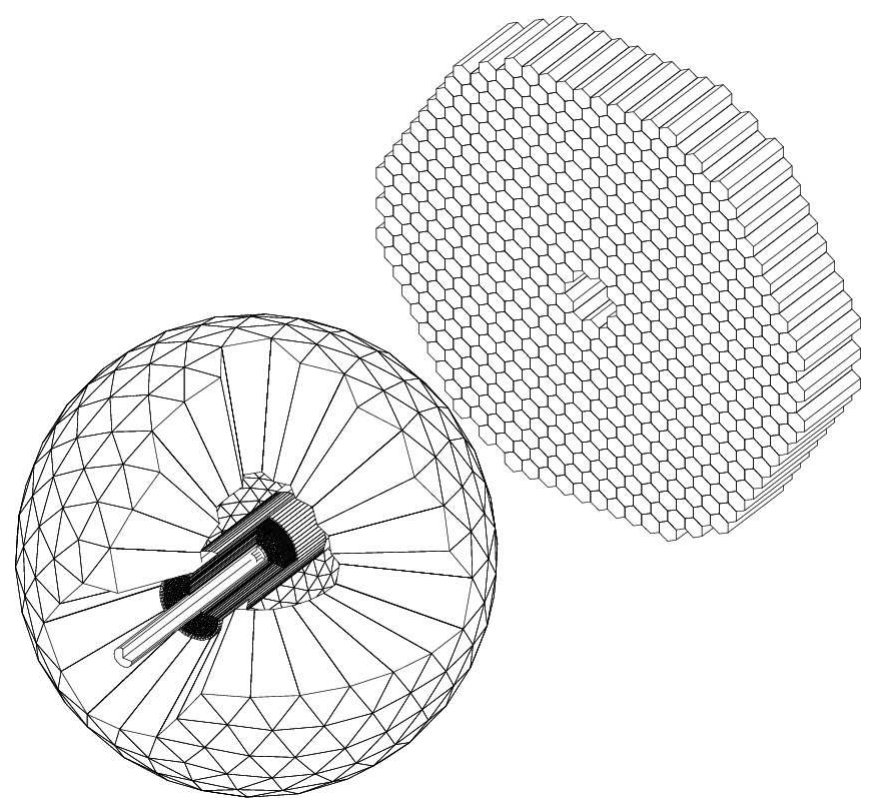

Fig. 26. Crystal Ball detector with inner tracker and TAPS forward wall.

step forward in the polarised target technology. The progression of the target polarisation measured during the experiment with the two target materials is shown in fig. 25 . The data analysis is in progress [37, 29,38].

\subsection{Conclusions and outlook}

The international GDH collaboration, working successfully at the electron accelerators MAMI and ELSA, has provided data to check the GDH sum rule experimentally for the first time. A newly developed polarised solid state target with high angular acceptance and highly polarisable new target materials in combination with $4 \pi$ detectors and highly polarised continuous photon beams made

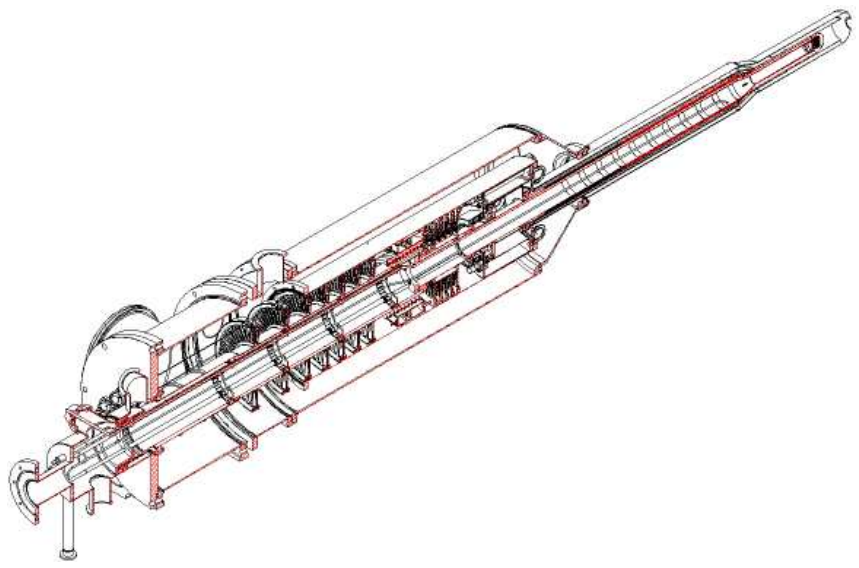

Fig. 27. The new dilution refrigerator for the Crystal Ball detector.

the experiment possible. For the proton the sum rule has been verified on a $10 \%$ level, the deuteron data are still being analysed. It is a challenge that requires a tremendous theoretical effort to extract the neutron properties from the deuteron data.

The investigation of the partial reaction channels led to an additional knowledge on the helicity dependence of the nucleon's excitation spectrum. The double-polarisation observable $E$ has been determined in a broad kinematical range.

With the upgrade of the MAMI accelerator to $1.5 \mathrm{GeV}$ a significant part of the outgoing particles would escape from the DAPHNE detector. The new standard detector to be used with the tagged photon beam, the Crystal Ball (CB) detector completed by TAPS as a forward wall, has already taken data in the years 2004 and 2005 with unpolarised targets, and both unpolarised and polarised photon beams. The CB consists of 672 optically isolated $\mathrm{NaI}(\mathrm{Tl})$ crystals, 15.7 radiation lengths thick. The counters are arranged in a spherical shell with an inner radius of $25.3 \mathrm{~cm}$ and an outer radius of $66.0 \mathrm{~cm}$. Charged particles can be measured by the central tracker consisting of a scintillator barrel and modified DAPHNE cylindrical multi-wire proportional chambers (two layers only). In order to achieve a better particle identification for charged particles, an inner barrel of plastic scintillators (PID-detector) has been included. The TAPS wall is composed of $522 \mathrm{BaF}_{2}$ detectors [39] arranged in a hexagon. The apparatus is schematically shown in fig. 26. The high granularity, large acceptance and good energy resolution make this setup a unique instrument for the detection of multi-photon final states.

The tagger will be upgraded to cope with the increased beam energy. Linearly and circularly polarised photons will be available. A polarised frozen-spin target is presently under development to allow for double polarisation experiments with the new facilities. The central part of this target will be a horizontal dilution refrigerator (see fig. 27).

The data presented in this paper have been mostly produced by the international GDH collaboration. We gratefully 
acknowledge the excellent support of the MAMI and ELSA accelerator groups. This work was supported by the Deutsche Forschungsgemeinschaft (SFB 201, SFB 443, Schwerpunktprogramm 1034, and GRK683), the INFN-Italy, the FWO Vlaanderen-Belgium, the IWT-Belgium, the UK Engineering and Physical Science Council, the DAAD, JSPS Research Fellowship, and the Grant-in-Aid (Specially Promoted Research) in Monbusho, Japan.

\section{References}

1. S.B. Gerasimov, Yad. Fiz. 2, 598 (1965) (Sov. J. Nucl. Phys. 2, 430 (1966)).

2. S.D. Drell, A.C. Hearn, Phys. Rev. Lett. 16, 908 (1966).

3. I. Karliner, Phys. Rev. D 7, 2717 (1973).

4. A. Sandorfi, Phys. Rev. D 50, R6681 (1994).

5. Anselmino et al., Sov. J. Nucl. Phys. 49, 553 (1989).

6. H. Olsen, L.C. Maximon, Phys. Rev. 114, 887 (1959).

7. C. Bradtke et al., Nucl. Instrum. Methods A 436, 430 (1999).

8. H. Dutz et al., Nucl. Instrum. Methods A 340, 272 (1994).

9. G. Audit et al., Nucl. Instrum. Methods A 301, 473 (1991).

10. J. Ahrens et al., Phys. Rev. Lett. 87, 022003 (2001).

11. J. Ahrens et al., Phys. Rev. Lett. 84, 5950 (2000).

12. O. Hansein et al., Nucl. Phys. A 632, 561 (1998).

13. R.A. Arndt et al., Phys. Rev. C 66, 055213 (2002).

14. D. Drechsel et al., Nucl. Phys. A 645, 145 (1999).

15. H. Dutz et al., Phys. Rev. Lett. 91, 192001 (2003).

16. H. Dutz et al., Phys. Rev. Lett. 93, 032003 (2004).
17. L. Tiator, Proceedings of GDH2002 (World Scientific, Singapore, 2003).

18. N. Bianchi, E. Thomas, Phys. Lett. B 450, 439 (1999).

19. S. Simula et al., Phys. Rev. D 65, 034017 (2002); private communication.

20. J. Ahrens et al., Eur. Phys. J. A 21, 323 (2004).

21. J. Ahrens et al., Phys. Rev. Lett. 88, 232002 (2002).

22. J. Ahrens et al., submitted to Phys. Lett. C.

23. J. Ahrens et al., Phys. Lett. B 551, 49 (2003).

24. J. Ahrens et al., Phys. Lett. B 624, 173 (2005).

25. J. Ahrens et al., Eur. Phys. J. A 17, 241 (2003).

26. D. Drechsel, Prog. Part. Nucl. Phys. 34, 181 (1995).

27. R. Beck, H.P. Krahn et al., Phys. Rev. Lett. 78 (1997).

28. G.S. Blanpied et al., Phys. Rev. Lett. 79, 4337 (1997).

29. T. Rostomyan, PhD Thesis (Gent) (2005).

30. J. Nacher, Proceedings of NSTAR2001 (World Scientific Pub. Co, 2001) p. 189.

31. M. Vanderhaeghen, H. Holvoet, private communication, Mainz 2001.

32. H. Arenhövel, The GDH for the deuteron, in Proceedings of GDH2000, Mainz, edited by D. Drechsel, L. Tiator (World Scientific, Singapore, 2001) p. 67.

33. A. Fix, private communication.

34. M. Schwamb, private communication.

35. M. Martinez, PhD Thesis, University of Mainz (in preparation).

36. St. Goertz et al., Nucl. Instrum. Methods A 526, 43 (2004).

37. O. Jahn, PhD Thesis, University of Mainz (2005).

38. S. McGee, PhD Thesis, Duke University (in preparation). 39. R. Novotny, IEEE Trans. Nucl. Sci. 38, 379 (1991). 